\title{
The universal minimal system for the group of homeomorphisms of the Cantor set
}

\author{
by \\ E. Glasner (Ramat Aviv) and B. Weiss (Jerusalem)
}

\begin{abstract}
Each topological group $G$ admits a unique universal minimal dynamical system $(M(G), G)$. For a locally compact noncompact group this is a nonmetrizable system with a rich structure, on which $G$ acts effectively. However there are topological groups for which $M(G)$ is the trivial one-point system (extremely amenable groups), as well as topological groups $G$ for which $M(G)$ is a metrizable space and for which one has an explicit description. We show that for the topological group $G=\operatorname{Homeo}(E)$ of self-homeomorphisms of the Cantor set $E$, with the topology of uniform convergence, the universal minimal system $(M(G), G)$ is isomorphic to Uspenskij's "maximal chains" dynamical system $(\Phi, G)$ in $2^{2^{E}}$. In particular it follows that $M(G)$ is homeomorphic to the Cantor set. Our main tool is the "dual Ramsey theorem", a corollary of Graham and Rothschild's Ramsey's theorem for $n$-parameter sets. This theorem is used to show that every minimal symbolic $G$-system is a factor of $(\Phi, G)$, and then a general procedure for analyzing $G$-actions of zero-dimensional topological groups is applied to show that $(M(G), G)$ is isomorphic to $(\Phi, G)$.
\end{abstract}

0. Introduction. This work is a sequel to our paper [3] and it establishes results similar to those obtained in $[3]$ for the group $\mathbb{S}(\mathbb{Z})$ (see also Pestov [7]). This time we are dealing with the Polish topological group $G=\operatorname{Homeo}(E)$ of self-homeomorphisms of the Cantor set $E$. More explicitly, we identify in the present work the universal minimal $G$-dynamical system $(M(G), G)$ as the natural induced action of $G$ on Uspenskij's "maximal chains space" $\Phi$ (a subset of the compact hyper-hyper-space $2^{2^{E}}$ ). In particular it follows that $M(G)$ is a compact metric space (in fact homeomorphic to the Cantor set) and can be described as the inverse limit of a countable collection of minimal symbolic $G$-systems. We also show that the system $(\Phi, G)$ is proximal, thereby establishing that every minimal $G$-system is necessarily proximal. Our methods of proof are similar to those employed in [3]. However, whereas in [3] the main combinatorial tool was the well known Ramsey theorem, here we use one of the consequences of Graham

2000 Mathematics Subject Classification: Primary 37B05; Secondary 37B10, 22-XX. 
and Rothschild's "Ramsey's theorem for $n$-parameter sets" as the key tool for proving dynamical results. It is perhaps worthwhile to point out the reversal of roles which takes place here: combinatorial results are employed for proving dynamical theorems rather than the reverse situation in which dynamical methods are utilized in order to prove (number-theoretical) combinatorial results - situations with which the Furstenberg school made us familiar in recent years.

The motivation for considering the question of identifying the universal minimal Homeo $(E)$-system becomes clear upon recalling some results established by V. Pestov and V. Uspenskij. First, Pestov [6] showed that the universal minimal dynamical system $(M(G), G)$ for the group $G=$ Homeo $_{+}\left(S^{1}\right)$, of orientation-preserving homeomorphisms of the circle with the compact-open topology, coincides with the natural action of $G$ on $S^{1}$. This provided the first nontrivial example of a metrizable universal minimal system. He then proposed the possibility that similarly for $G=\operatorname{Homeo}(X)$, the group of homeomorphisms of any compact manifold $X$ (say a sphere), the universal minimal $G$-system coincides with the natural action of $G$ on $X$. Then, in [9], V. Uspenskij showed that the action of a topological group $G$ on its universal minimal system $M(G)$ is never 3-transitive, so that, e.g., for manifolds $X$ of dimension $>1$ as well as for $X=Q$, the Hilbert cube, and $X=E$, the Cantor set, $M(G)$ cannot coincide with $X$. To establish this result he constructed, for any compact dynamical system, the associated dynamical system $\Phi$ of maximal chains, which in the case of the canonical action of $G=\operatorname{Homeo}(E)$ on $E$ turns out to be isomorphic to the universal minimal system $(M(G), G)$. We refer to the introduction to our previous paper [3] for further motivation and background concerning the study of universal minimal systems.

We thank Noga Alon for confirming the validity of the relevant combinatorial result, which our investigation of the dynamical problem led us to formulate. Later we found it mentioned explicitly in [8] where it is called the "dual Ramsey theorem".

1. The space $\Phi(E)$ of maximal chains. For a compact space $Y$, given a finite collection of open sets $\mathcal{U}=\left\{U_{1}, \ldots, U_{k}\right\}$ in $Y$, we set

$$
\left\langle U_{1}, \ldots, U_{k}\right\rangle=\left\{F \in 2^{E}: \forall j, F \cap U_{j} \neq \emptyset \text { and } F \subset \bigcup_{j=1}^{k} U_{j}\right\} .
$$

The family of sets of the form (1.1) forms a basis for the Vietoris topology on $2^{Y}$, the space of closed subsets of $Y$. Let $E$ be the Cantor set and let $G$ denote the Polish group of self-homeomorphisms of $E$ equipped with the topology of uniform convergence. The Cantor set $E$ is characterized, 
up to a homeomorphism, by being compact, separable, perfect and zerodimensional. However, we will consider it here as the classical Cantor set, i.e. the ternary subset of the interval $[0,1]$. Thus $E$ has the following representation:

$$
E=\bigcap_{n=0}^{\infty} I^{n},
$$

where $I^{n}=\bigcup_{j=1}^{2^{n}} I_{j}^{n}$ is the disjoint union of the $2^{n}$ closed intervals obtained by removing from $I=[0,1]$ the appropriate $2^{n}-1$ open "middle third" intervals, which we call the adjacent intervals. In what follows we will write $I_{j}^{m}$ for the clopen subset $I_{j}^{m} \cap E$ of $E$. For each integer $m \geq 1$, $\mathbf{I}^{m}=\left\{I_{j}^{m}: 1 \leq j \leq 2^{m}\right\}$ denotes the basic partition of $E$ into $2^{m}$ clopen "intervals".

Let $\Phi=\Phi(E) \subset 2^{2^{E}}$ be the collection of maximal chains on $E$. Recall that a chain on $E$ is a family $c=\left\{F_{\alpha}\right\}_{\alpha \in A}$ of closed subsets linearly ordered by inclusion (for $F_{\alpha}, F_{\beta} \in c$ either $F_{\alpha} \subset F_{\beta}$ or $F_{\beta} \subset F_{\alpha}$ ). A chain $c$ is called maximal if it is maximal with respect to inclusion (if $c \subset c^{\prime}$ and $c^{\prime}$ is a chain then $c=c^{\prime}$ ). As was shown by Uspenskij (see [9]), $\Phi$ is a closed subset of the compact and metrizable space $2^{2^{E}}$. The natural action of $G$ on $E$ induces an action of $G$ on $2^{2^{E}}$, and with respect to this action $\Phi$ is an invariant set. Clearly every $c \in \Phi$ has a first element of the form $\{r(c)\}$ with $r(c) \in E$. It is easy to verify that the root map $r:(\Phi, G) \rightarrow(E, G)$ is a homomorphism of dynamical systems.

For each permutation $\alpha \in S_{2^{n}}$ and $1 \leq k \leq 2^{n}$ let

$$
\mathcal{J}_{\alpha}^{k}=\left\langle I_{\alpha(1)}^{n}, \ldots, I_{\alpha(k)}^{n}\right\rangle \text {. }
$$

Thus $\mathrm{J}_{\alpha}^{k}$ is a clopen subset of $2^{E}$ and we now set

$$
\begin{aligned}
\mathcal{U}_{\alpha}^{n} & =\left\langle\mathcal{J}_{\alpha}^{1}, \mathcal{J}_{\alpha}^{2}, \ldots, \mathcal{J}_{\alpha}^{2^{n}}\right\rangle \cap \Phi \\
& =\left\langle\left\langle I_{\alpha(1)}^{n}\right\rangle,\left\langle I_{\alpha(1)}^{n}, I_{\alpha(2)}^{n}\right\rangle, \ldots,\left\langle I_{\alpha(1)}^{n}, I_{\alpha(2)}^{n}, \ldots, I_{\alpha\left(2^{n}\right)}^{n}\right\rangle\right\rangle \cap \Phi \\
& =\left\{c \in \Phi: \forall j \exists F \in c \cap \mathcal{J}_{\alpha}^{j}, \text { and } \forall F \in c \exists j, F \in \mathcal{J}_{\alpha}^{j}\right\} .
\end{aligned}
$$

The proof of the following lemma is straightforward.

LEMma 1.1. The collection $\left\{\mathcal{U}_{\alpha}^{n}: \alpha \in \bigcup_{n=1}^{\infty} S_{2^{n}}\right\}$ is a basis for the Vietoris topology on $\Phi \subset 2^{2^{E}}$.

Let $P \subset G$ be the group of order preserving homeomorphisms of $E$. With every $p \in P$ we associate the unique homeomorphism $\tilde{p}:[0,1] \rightarrow[0,1]$ such that $\tilde{p}\lceil E=p$ and $\widetilde{p}$ is linear on the adjacent open intervals. The map $p \mapsto \widetilde{p}$ is a topological isomorphism of the group $P$ onto the closed subgroup $\widetilde{P}=\{\widetilde{p}: p \in P\}$ of the Polish group $\operatorname{Aut}(I,<)$ of order preserving homeomorphisms of $I=[0,1]$. 
THEOREM 1.2. The topological group $P$ is topologically isomorphic to the Polish group $\operatorname{Aut}(\mathbb{Q},<)$ of order preserving bijections of the rational numbers $\mathbb{Q}$, equipped with the topology of pointwise convergence. In particular then, $P$ has the fixed point property on compacta.

Proof. Let $\left\{V_{n}\right\}_{n \in \mathbb{N}}$ be some enumeration of the set of adjacent open intervals associated with the construction of $E$. The order induced on the collection of these intervals by the natural order on $[0,1]$ has the order type of the rational numbers $\mathbb{Q}$ (it is linear, there is no first nor last element and there are infinitely many intervals between any two). It is easy to check that any element $p \in P$ defines an element $A(p)=\alpha_{p} \in \operatorname{Aut}(\mathbb{Q},<)$ and that the map $p \mapsto A(p), p \in P$, is a topological isomorphism of $P$ onto $\operatorname{Aut}(\mathbb{Q},<)$. By a theorem of Pestov $[6]$ the group $\operatorname{Aut}(\mathbb{Q},<)$ has the fixed point property on compacta and therefore so does $P$.

For $t \in[0,1]$ let $E_{t}=E \cap[0, t]$ and set $c_{0}=\left\{E_{t}: t \in[0,1]\right\}$. It is easy to check that $c_{0}$ is a maximal chain on $E$, i.e. an element of $\Phi$. Two more examples of chains with roots at $0 \in E$ are the chains $c_{0}^{+}=$ $\{\{0\}\} \cup\left\{E_{t} \cup\{1\}: t \in[0,1]\right\}$ and $c_{0}^{-}=\{\{0\}\} \cup\left\{F_{t} \cup\{0\}: t \in[0,1]\right\}$, where $F_{t}=E \cap[t, 1]$. We let $c_{1}, c_{1}^{ \pm}$be the images of the chains $c_{0}, c_{0}^{ \pm}$under the homeomorphism $\sigma \in G, \sigma: E \rightarrow E$ obtained by restricting the reflection of $[0,1]$ about its midpoint $1 / 2$ to $E$. Note that for any dense sequence of distinct points in $E$, say $\left\{x_{0}, x_{1}, \ldots\right\}$, the ascending sequence of finite sets $c=\left\{\left\{x_{0}, x_{1}, \ldots, x_{n}\right\}: n=0,1, \ldots\right\} \cup\{\{E\}\}$ forms an element of $\Phi$ with root $r(c)=x_{0}$.

THEOREM 1.3. (i) The action of $G$ on $\Phi$ is minimal.

(ii) The stability subgroup $\mathrm{St}_{c_{0}}=\left\{g \in G: g c_{0}=c_{0}\right\}$ coincides with $P$.

(iii) The six points $\left\{c_{0}, c_{0}^{ \pm}, c_{1}, c_{1}^{ \pm}\right\}$are the only fixed points of $P$ in $\Phi$.

(iv) The action of $G$ on $\Phi$ is proximal.

Proof. (i) Given two basic neighborhoods of the same "rank" $\mathcal{U}_{\alpha}^{n}$ and $\mathcal{U}_{\beta}^{n}$, $\alpha, \beta \in S_{2^{n}}$, as given in Lemma 1.1, there exists a homeomorphism $h \in G$ which maps one onto the other, e.g. the obvious piecewise linear homeomorphism associated with the permutation $\alpha \circ \beta^{-1}$. This property clearly implies minimality of $(\Phi, G)$.

(ii) For every $p \in P$ and $t \in[0,1]$ we have $p\left(E_{t}\right)=E_{p(t)}$ and it follows that $p c_{0}=\left\{p\left(E_{t}\right): t \in[0,1]\right\}=c_{0}$. Conversely, if $g c_{0}=c_{0}$ for $g \in G$ then for every $t \in E, g\left(E_{t}\right)=E_{s}$ for some $s \in E$. The fact that $c_{0}$ is a chain implies that $s=g(t)$, and that $g$ is in $P$.

(iii) Clearly each of these six points is a fixed point for $P$. Conversely, assume that $c \in \Phi$ is $P$-fixed with $r(c)=0$. We first observe that elements $F \in c$ can have no nontrivial "gaps". In fact, if $F \in c$ is such that for some real numbers $0<a<b<1$ we have $E \cap(a, b) \neq \emptyset, F \cap(a, b)=\emptyset$ yet 
$F \cap(0, a] \neq \emptyset$ as well as $F \cap[b, 1) \neq \emptyset$, then there surely is a homeomorphism $p \in P$ with $F \backslash p F \neq \emptyset \neq p F \backslash F$. However, as $p c=c$, we also have $F, p F \in c$. Since these two sets are not comparable this contradicts the fact that $c$ is a chain. We observe that this immediately rules out the possibility that the chain $c$ is of the "discrete type" described above. It follows that for every $F \in c$ with $\{0\} \varsubsetneqq F \varsubsetneqq E$, there exists $0<a<1$ such that either $F \cap[0, a)$ or $F \cap(a, 1]$ is an infinite set. In the first case it is then easy to verify that for every $t \in[0,1]$ there exists a sequence $p_{n} \in P$ such that either $\lim p_{n} F=E_{t}$ or $\lim p_{n} F=E_{t} \cup\{1\}$ (in the compact space $2^{E}$ ). Since $p_{n} c=c$ for each $n$, it follows that either $E_{t}$ or $E_{t} \cup\{1\}$ is in $c$. It is now clear how one finishes the proof.

(iv) Let $c_{1}, c_{2}$ be two chains in $\Phi$. Let $Z=\operatorname{cls}\left\{\left(g c_{1}, g c_{2}\right): g \in G\right\}$ be the orbit closure of the point $\left(c_{1}, c_{2}\right)$ in the product system $(\Phi \times \Phi, G)$. By the fixed point property of $P$, there exists a point $\left(a_{1}, a_{2}\right) \in Z$ with $p a_{i}=a_{i}$ for every $p \in P, i=1,2$. By (iii) we know that both $a_{1}$ and $a_{2}$ are in $\left\{c_{0}, c_{0}^{ \pm}, c_{1}, c_{1}^{ \pm}\right\}$. It therefore suffices to show that any pair of points in this set is proximal. Let us consider the case $a_{1}=c_{0}$ and $a_{2}=c_{1}$. Let $\mathcal{U}_{\mathrm{id}}^{m}$ be the basic neighborhood of $c_{0}$ at level $m$. By considering the canonical partition $\left\{I_{j}^{n}: j=1, \ldots, 2^{n}\right\}$ of a much higher order $n$, it is easy to "cook up" a homeomorphism $g \in G$ that will map the first $2^{m}$ and the last $2^{m}$ intervals of this partition into the $2^{m}$ corresponding intervals $\left\{I_{j}^{m}: j=1, \ldots, 2^{n}\right\}$ in their natural order. For such $g$ we have $g c_{0}, g c_{1} \in \mathcal{U}_{\text {id }}^{m}$ and we conclude that indeed $c_{0}$ and $c_{1}$ are proximal. The arguments proving the proximality of the other pairs are similar and we omit the proofs.

2. On the structure of the group $G=\operatorname{Homeo}(E)$. For each $n$ we let

$$
H_{n}=\left\{g \in G: g\left(I_{j}^{n}\right)=I_{j}^{n}, \forall 1 \leq j \leq 2^{n}\right\}
$$

Clearly $H_{n}$ is a clopen subgroup of $G$ and we note that the system of clopen subgroups $\left\{H_{n}: n=2,3, \ldots\right\}$ forms a basis for the topology of $G$ at the identity $e \in G$. The results in this section are not required for the proof of our main result. We include them for completeness.

TheOREM 2.1. Let $L$ be the subset of $G$ consisting of all homeomorphisms $g \in G$ which preserve $\lambda$, the Cantor-Lebesgue measure on $E$. Then $L$ and $P$ are closed subgroups of $G, L \cap P=\{$ id $\}$ and $G=L P=P L$.

Proof. Let $g \in G$ be given and set $\nu=g \lambda$. For every $I_{j}^{n}$ we have $\nu\left(I_{j}^{n}\right)=\lambda\left(g^{-1} I_{j}^{n}\right)$ and it follows that to each number in the set $\{\nu([0, t])$ : $E_{t}=E \cap[0, t]$ is a clopen subset of $\left.E\right\}$ corresponds a unique $p(t) \in E$ such that $E_{p(t)}=[0, p(t)] \cap E$ is clopen and $\lambda([0, t])=\nu([0, p(t)])$. The map $p$ extends uniquely to a homeomorphism $p \in G$ and we have $p^{-1} g \lambda([0, t])=$ 
$g \lambda([0, p(t)])=\nu([0, p(t)])=\lambda([0, t])$. Thus $l=p^{-1} g \in L$ and $g=p l$. The relation $L \cap P=\{$ id $\}$ is easy to verify.

It is also easy to verify that in the unique decomposition $h=l p$ of an element $h \in H_{n}$ as a product of $l \in L$ and $p \in P$ we have $l \in L_{n}=L \cap H_{n}$ and $p \in P_{n}=P \cap H_{n}$, i.e. $H_{n}=L_{n} P_{n}$. Similarly $H_{n}=P_{n} L_{n}$.

The next proposition shows that a direct proof of the universality of $(\Phi, G)$ along the lines of Pestov's proof in [7] of the main result of [3] (i.e. by showing that $G / P$ uniformly embeds into $(M(G), G))$ is not possible.

Proposition 2.2. (i) The natural projection $\pi: G \rightarrow G / P$ restricts to a homeomorphism onto $\pi: L \rightarrow G / P$.

(ii) The map $g P \mapsto g c_{0}$ from $G / P$ onto the $G$-orbit $G c_{0} \subset \Phi$ is 1-1 and continuous but not a homeomorphism.

Proof. (i) Clearly the restriction $\pi: L \rightarrow G / P$ is $1-1$, continuous and onto. A basic open neighborhood of $g \in G$ has the form $U=g H_{n}$ for some $n \geq 0$. Clearly for $g \in L, L \cap U=g\left(L \cap H_{n}\right)=g L_{n}$ and therefore the sets $g L_{n}, n \in \mathbb{N}$, form a basis for the relative topology at $g \in L$. Now $\pi\left(g L_{n}\right)=g L_{n} P=g L_{n} P_{n} P=g H_{n} P=\pi\left(g H_{n}\right)$ and as the latter is open in $G / P$ we see that $\pi: L \rightarrow G / P$ is also open, hence a homeomorphism.

(ii) If we consider the commutative diagram

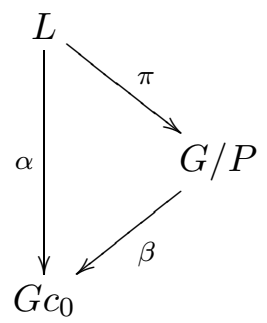

we have $\alpha=\beta \circ \pi$. Since the maps $\pi$ and $\alpha$ are continuous, so is $\beta$. Now it is not hard to construct a sequence $l_{n} \in L$ such that $l_{n} c_{0} \rightarrow c_{0}$ in $\Phi$ but $l_{n} \not t e$ in $L$ so that $\beta$ is not a homeomorphism.

LEMma 2.3. The closed subgroup $P \subset G$ has the following properties:

(i) $P \subset g P g^{-1} \Rightarrow g \in P \cup \sigma P$.

(ii) $\bigcap_{g \in G} g^{-1} P g=\{e\}$.

Proof. (i) We have

$$
P g c_{0} \subset\left(g P g^{-1}\right) g c_{0}=g P c_{0}=g c_{0},
$$

hence, by Theorem 1.3(iii), $g c_{0}$ is an element of the set $\left\{c_{0}, c_{0}^{ \pm}, c_{1}, c_{1}^{ \pm}\right\}$of $P$-fixed points in $\Phi$. Clearly then $g c_{0} \in\left\{c_{0}, c_{1}\right\}$ and we either have $g c_{0}=c_{0}$, in which case $g \in P$, or $g c_{0}=\sigma c_{0}$ and then $\sigma g \in P$.

(ii) For $h \in \bigcap_{g \in G} g^{-1} P g$ we have $h g c_{0}=g c_{0}$ for every $g \in G$. Thus $h$ acts as the identity on a dense subset of $\Phi$, hence on all of $\Phi$. In particular 
$h r(c)=r(c)$ for every $c \in \Phi$ and we conclude that $h$ acts as the identity on $E$; i.e. $h=\mathrm{id}=e$.

3. The symbolic dynamical systems $\left(\Omega^{k}, G\right)$. Fix an integer $k \geq 1$ and consider the collection

$$
\mathcal{A}^{k}=\left\{\mathbf{a}=\left\{A_{1}, \ldots, A_{k}\right\} \text { : a partition of } E \text { into } k \text { nonempty clopen sets }\right\} .
$$

As above we let, for each integer $m \geq 1, \mathbf{I}^{m}=\left\{I_{j}^{m}: 1 \leq j \leq 2^{m}\right\}$ denote the basic partition of $E$ into $2^{m}$ clopen "intervals".

Each $\mathbf{a} \in \mathcal{A}^{k}$ determines a smallest integer $m^{\prime}$, with $2^{m^{\prime}} \geq k$, such that $\mathbf{a}$ is $\mathbf{I}^{m^{\prime}}=\left\{I_{j}^{m^{\prime}}: 1 \leq j \leq 2^{m^{\prime}}\right\}$ measurable. We denote by $\mathcal{A}_{m}^{k}$ the (finite) collection of all a whose $m^{\prime} \leq m$. Thus $\mathcal{A}^{k}=\bigcup_{m \geq 1} \mathcal{A}_{m}^{k}$. It will be convenient to identify elements of $\mathcal{A}_{m}^{k}$ as labelling with exactly $k$ labels of the collection $\mathbf{I}^{m}$. Thus

$$
\mathcal{A}_{m}^{k} \cong\{1, \ldots, k\}_{*}^{2^{m}}
$$

where the $*$ means that only ordered sequences (of length $2^{m}$ ) where all $k$ digits appear are counted.

For a fixed partition $\left\{B_{1}, \ldots, B_{k}\right\}$ of $E$ into $k$ nonempty clopen sets, let

$$
H=\left\{g \in G: g B_{j}=B_{j}, \forall 1 \leq j \leq k\right\} .
$$

Then $H$ is a clopen subgroup and the discrete homogeneous space $H \backslash G$ can be identified with $\mathcal{A}^{k}$. In fact an element $H g \in H \backslash G$ is uniquely determined by the partition $\mathbf{a}=\left\{g^{-1} B_{j}: 1 \leq j \leq k\right\}$, and conversely to every partition $\mathbf{a} \in \mathcal{A}^{k}$ corresponds a coset $H g \in H \backslash G$. In fact, if $\mathbf{a}=\left\{A_{1}, \ldots, A_{k}\right\}$ we can choose $g$ to be any homeomorphism of $E$ with $A_{j}=g^{-1} B_{j}$.

In particular for $k=2^{n}$, taking $B_{j}=I_{j}^{n}, 1 \leq j \leq 2^{n}$, we have a parameterization of $\mathcal{A}^{k}=\mathcal{A}^{2^{n}}$ by the discrete homogeneous space $H_{n} \backslash G$.

Set

$$
\Omega^{k}=\{1,-1\}^{\mathcal{A}^{k}} \cong\{1,-1\}^{H_{n} \backslash G} .
$$

The group $G$ acts on the compact space $\Omega^{k}$ as follows. For $\omega \in \Omega^{k}$ and $g \in G$ let

$$
g \omega(\mathbf{a})=\omega\left(g^{-1} A_{1}, \ldots, g^{-1} A_{k}\right)
$$

for any $\mathbf{a}=\left\{A_{1}, \ldots, A_{k}\right\} \in \mathcal{A}^{k}$, and the $G$-action on $\Omega^{k}=\Omega^{2^{n}}$ can be described equivalently by $g \omega\left(H_{n} g^{\prime}\right)=\omega\left(H_{n} g^{\prime} g\right)$ for every $H_{n} g^{\prime} \in H_{n} \backslash G$.

Again for $k=2^{n}$ let $S_{k}$ denote the group of permutations of $\{1, \ldots, k\}$ and set

$$
\mathcal{T}^{k}=\{1,-1\}^{S_{k}}
$$

We call elements of $\mathcal{T}^{k}$ tables. The natural action of $S_{k}$ on $\{1, \ldots, k\}$ defines 
induced actions of $S_{k}$ on $\mathcal{T}^{k}, \mathcal{A}^{k}$ and $\Omega^{k}$ as follows. For $\sigma, \theta \in S_{k}, T \in \mathcal{T}^{k}$, $\mathbf{a}=\left\{A_{1}, \ldots, A_{k}\right\} \in \mathcal{A}^{k}$ and $\omega \in \Omega^{k}$ let

$$
\left(L_{\sigma} T\right)(\theta)=T\left(\sigma^{-1} \theta\right), \quad \sigma \mathbf{a}=\left\{A_{\sigma^{-1}(1)}, \ldots, A_{\sigma^{-1}(k)}\right\}, \quad(\sigma \omega)(\mathbf{a})=\omega(\sigma \mathbf{a}) .
$$

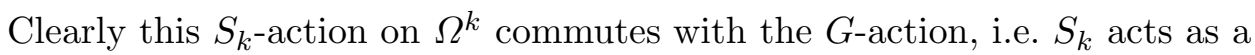
group of automorphisms of the dynamical system $\left(\Omega^{k}, G\right)$.

Using tables we can give an alternative description of elements of $\Omega^{k}=$ $\{1,-1\}^{\mathcal{A}^{k}}$. Let $\widehat{\mathcal{A}}^{k}$ be the quotient space $\mathcal{A}^{k} / S_{k}$, i.e. the collection of unordered partitions of $E$ into $k$ nonempty clopen sets. Given $\omega \in \Omega^{k}$ let $\widehat{\omega} \in \widehat{\Omega}^{k}=\left(\mathcal{T}^{k}\right)^{\widehat{\mathcal{A}}^{k}}$ be defined by

$$
(\widehat{\omega})(\widehat{\mathbf{a}})(\sigma)=\omega\left(\sigma \mathbf{a}^{*}\right),
$$

where $\mathbf{a}^{*}$ is obtained from the partition $\mathbf{a}=\left\{A_{1}, \ldots, A_{k}\right\} \in \mathcal{A}^{k}$ by rearranging the sets $A_{j}$ in the order in which they appear on $E$ going from left to right.

It is easy to see that $\omega \mapsto \widehat{\omega}$ is an isomorphism of the $G$-system $\left(\Omega^{k}, G\right)$ onto the $G$-system $\left(\widehat{\Omega}^{k}, G\right)$, where the $G$-action on the latter is defined by $(g \widehat{\omega})(\widehat{\mathbf{a}})=(\widehat{\omega})\left(g^{-1} \widehat{\mathbf{a}}\right)$.

4. The symbolic factors of $(\Phi, G)$. In this section we show that all the minimal subsets of the system $\left(\Omega^{k}, G\right)$ are factors of $(\Phi, G)$. Throughout the section $k=2^{n}$. We first indicate how one associates with each table $T \in \mathcal{T}^{k}$ a minimal subsystem $\Sigma_{T} \subset \Omega^{k}$ and a homomorphism $\phi_{T}:(\Phi, G) \rightarrow\left(\Sigma_{T}, G\right)$.

Let $U_{T} \subset \Phi$ be the clopen subset

$$
\bigcup\left\{\mathcal{U}_{\alpha}^{n}: \alpha \in S_{k}, T(\alpha)=1\right\} .
$$

We let $\phi_{T}: \Phi \rightarrow \Omega^{k}$ be the associated "name map". As indicated in Section 3 we identify $\mathcal{A}^{k}$ with $H_{n} \backslash G$ and set

$$
\phi_{T}(c)\left(H_{n} g\right)= \begin{cases}1, & g c \in U_{T}, \\ -1, & g c \notin U_{T} .\end{cases}
$$

It is easily seen that $\phi_{T}$ is indeed a homomorphism from $(\Phi, G)$ into $(\Omega, G)$ and we set $\Sigma_{T}=\phi_{T}(\Phi)$.

TheOREM 4.1. Every minimal subset of the system $\left(\Omega^{k}, G\right)$ is a factor of the minimal system $(\Phi, G)$.

Proof. Fix a minimal subset $\Sigma \subset \Omega^{k}$. We shall construct a homomorphism $\phi: \Phi \rightarrow \Sigma$. Fix a point $\omega \in \Sigma$.

As described in Section 3, the corresponding element $\widehat{\omega} \in \widehat{\Omega}^{k}=\left(\mathcal{T}^{k}\right)^{\widehat{\mathcal{A}}^{k}}$ is defined by 


$$
(\widehat{\omega})(\widehat{\mathbf{a}})(\sigma)=\omega\left(\sigma \mathbf{a}^{*}\right),
$$

where $\mathbf{a}^{*}$ is obtained from the partition $\mathbf{a}=\left\{A_{1}, \ldots, A_{k}\right\} \in \mathcal{A}^{k}$ by rearranging the sets $A_{j}$ in the order in which they appear on $E$ going from left to right. We consider $\widehat{\omega}$ as a coloring of elements of $\widehat{\mathcal{A}}^{k}$ (i.e. unordered $k$-partitions) by $r$ "colors", where the colors we use are the tables in $\mathcal{T}^{k}=$ $\{1,-1\}^{S_{k}}$, so that $r=\operatorname{card} \mathcal{T}^{k}=2^{k !}$.

Applying the dual Ramsey theorem (Corollary 10 of [4], see also Corollary 4.2 of [8], page 130), for each $m$ there exists a large number $n=$ $D R(k, m, r)$ and a partition of $\left\{0,1, \ldots, 2^{n}-1\right\}$ into $2^{m}$ sets so that when inducing a coloring on $\widehat{\mathcal{A}}_{m}^{k}$ from the coloring defined by $\widehat{\omega}\left\lceil\widehat{\mathcal{A}}_{n}^{k}\right.$ we get a monochromatic coloring.

This means that applying a suitable $g_{m} \in G$ to $\omega$, we get an $\omega_{m}=g_{m} \omega$ in the $G$-orbit of $\omega$ which has a constant table on $\widehat{\mathcal{A}}_{m}^{k}$.

Taking a subsequence $m_{j}$ so that $\lim _{j \rightarrow \infty} \omega_{m_{j}}=\omega_{0}$ exists we find that $\omega_{0}$ is in the minimal set $\Sigma$ and that as an element of $\Omega^{k}$ it has a constant table $T$. Clearly $\phi_{T}\left(c_{0}\right)=\omega_{0}$ and we conclude that $\Sigma=\Sigma_{T}$ as required.

\section{The universal transitive and minimal systems of a topological} group $T$. This section is reproduced verbatim from [3] in order to make the exposition self-contained.

Let $T$ be a topological group; we write $\mathcal{L}(T)$ for the commutative $C^{*}$ algebra of bounded left uniformly continuous $\mathbb{C}$-valued functions on $T$ with the norm $\|f\|=\sup _{t \in T}|f(t)|$, and with $f^{*}(t)=\overline{f(t)}$. Recall that a function $f: T \rightarrow \mathbb{C}$ is in $\mathcal{L}(T)$ iff it is bounded and for every $\varepsilon>0$ there exists a symmetric neighborhood $V=V^{-1}$ of the unit element $e \in T$ with

$$
s t^{-1} \in V \Rightarrow|f(s)-f(t)|<\varepsilon .
$$

An equivalent condition is $\left\|L_{r} f-f\right\|<\varepsilon$ for every $r \in V$, where $L_{r} f(t)=$ $f\left(r^{-1} t\right)$. It is easy to see that $\mathcal{L}(T)$ is right and left $T$-invariant; that is, $f \in \mathcal{L}(T) \Rightarrow R_{s} f \in \mathcal{L}(T)$ and $L_{s} f \in \mathcal{L}(T)$, where $R_{s} f(t)=f(t s)$ and $L_{s} f(t)=f\left(s^{-1} t\right)$. The next lemma is well known and its proof is straightforward.

Lemma 5.1. Let $\left(X, x_{0}, T\right)$ be a pointed T-dynamical system (i.e. $X$ is a compact Hausdorff space and the action $(t, x) \mapsto t x, T \times X \rightarrow X$ is jointly continuous; $x_{0} \in X$ is a distinguished point with $\left.\overline{\mathcal{O}}_{T}\left(x_{0}\right)=X\right)$. Let $F \in C(X)$. Then the function $f=f_{x_{0}}$ defined by $f_{x_{0}}(t)=F\left(t x_{0}\right)$ is an element of $\mathcal{L}(T)$. In fact the map

$$
\Phi: F \mapsto f_{x_{0}}, \quad \Phi: C(X) \rightarrow \mathcal{L}(T)
$$


is a linear isometry of $C^{*}$-algebras such that for every $s \in T$,

$$
\Phi \circ L_{s}=L_{s} \circ \Phi .
$$

In the situation described in the lemma, we say that the function $f$ is coming from the pointed system $\left(X, x_{0}, T\right)$.

Let $\mathbf{L}$ be the Gelfand space corresponding to the $C^{*}$-algebra $\mathcal{L}(T)$ and $\ell_{0} \in \mathbf{L}$ the multiplicative functional $\ell_{0}: f \mapsto f(e)$ corresponding to the evaluation of a function in $\mathcal{L}(T)$ at the identity element $e \in T$.

COROLlary 5.2. With the natural action of the group $T$ on the Gelfand space $\mathbf{L}$ and the distinguished point $\ell_{0}$, the pointed dynamical system $\left(\mathbf{L}, x_{0}, T\right)$ is the universal point transitive T-system. That is, for every point transitive $T$-system $\left(X, x_{0}, T\right)$ with distinguished transitive point $x_{0}$ there exists a unique homomorphism

$$
\phi:\left(\mathbf{L}, \ell_{0}, T\right) \rightarrow\left(X, x_{0}, T\right) .
$$

Proof. The map $\phi$ is realized by the dual of the isometric isomorphism $\Phi: C(X) \rightarrow \mathcal{L}(T)$ on the corresponding Gelfand spaces.

We shall use the notation $|\mathcal{A}|$ for the Gelfand space of a closed $T$-invariant subalgebra $\mathcal{A} \subset \mathcal{L}(T)$ (i.e. $L_{s} \mathcal{A}=\mathcal{A}$ for every $s \in T$ ). Thus with this notation $\mathbf{L}=|\mathcal{L}(T)|$ and in the above corollary $|\mathcal{A}| \cong X$ where $\mathcal{A}=\Phi(C(X))$.

Let now $\mathbf{M} \subset \mathbf{L}$ be any minimal subset. If $(X, T)$ is a minimal system then the restriction of the map $\phi: \mathbf{L} \rightarrow X$ to $\mathbf{M}$ is a homomorphism $\phi: \mathbf{M} \rightarrow X$. So in this sense $\mathbf{M}$ is a universal minimal system. It turns out that in fact any two minimal sets $\mathbf{M}_{1}$ and $\mathbf{M}_{2}$ of $\mathbf{L}$ are isomorphic as dynamical systems (we shall not prove this fact here; see for example [1]). Thus $(\mathbf{M}, T)$ is the unique universal minimal system (although not as a pointed system; fixing a distinguished point $m_{0} \in \mathbf{M}$ and given a pointed minimal system $\left(X, x_{0}, T\right)$, a homomorphism $\phi: \mathbf{L} \rightarrow X$ with $\phi\left(m_{0}\right)=x_{0}$ may not exist). The next theorem is due to Pestov [6] (see also [5]).

THEOREM 5.3. If the topology of $T$ admits a basis for neighborhoods at $e$ consisting of clopen subgroups, then the topological space $\mathbf{L}$ (and hence also $\mathbf{M}$ ) is zero-dimensional.

Proof. Given a clopen subgroup $H \subset T$ let

$$
\mathcal{L}_{H}=\left\{f \in \mathcal{L}(T): L_{s} f=f, \forall s \in H\right\} .
$$

If $\tilde{f}$ is any bounded function on the discrete space $H \backslash T$ then the corresponding lift $f(t)=\widetilde{f}(H t)$ is an element of $\mathcal{L}_{H}$ and conversely every element of $\mathcal{L}_{H}$ defines a function in $l^{\infty}(H \backslash T)$. Thus $\left|\mathcal{L}_{H}\right| \cong \beta(H \backslash T)$ where $\beta(H \backslash T)$ is the Stone-Cech compactification of the discrete space $H \backslash T$; in particu$\operatorname{lar}\left|\mathcal{L}_{H}\right|$ is zero-dimensional (in fact extremely disconnected when $H \backslash T$ is infinite; see [2]). 
Given $f \in \mathcal{L}(T)$ and $\varepsilon>0$ there exists a clopen subgroup $H \subset T$ with $\sup _{t \in T} \operatorname{diam} f(H t)<\varepsilon$. The function $\widetilde{g} \in l^{\infty}(H \backslash T)$ defined by $\widetilde{g}(H t)=$ $\inf \{f(r t): r \in H\}$ lifts to a function $g(t)=\widetilde{g}(H t)$ in $\mathcal{L}_{H}$ with $\|g-f\| \leq \varepsilon$.

It follows that the algebra

$$
\bigcup\left\{\mathcal{L}_{H}: H \text { is a clopen subgroup of } T\right\}
$$

is dense in $\mathcal{L}(T)$ and by the Stone-Weierstrass theorem, its closure is all of $\mathcal{L}(T)$. We conclude that

$$
\mathbf{L}=\lim _{\longleftarrow}\left|\mathcal{L}_{H}\right|
$$

is the inverse limit of the zero-dimensional spaces $\left|\mathcal{L}_{H}\right|$ over the directed system of clopen subgroups $H \subset T$. In particular we conclude that $\mathbf{L}$ and therefore also its subset $\mathbf{M}$ are zero-dimensional.

Again let $(X, T)$ be a $T$-dynamical system and let $F \in C(X)$ be a realvalued function. Let $I$ be the interval $[-\|F\|,\|F\|]$, and consider the compact space $I^{T}$ of all maps from $T$ to $I$ (with the topology of pointwise convergence). We define a map $\psi: X \rightarrow I^{T}$ by $\psi(x)=f_{x}$, i.e. $f_{x}(t)=F(t x)$ $(t \in T)$. Let $Y=\psi(X)$. As observed in Lemma 5.1 each $f_{x}$ is in $\mathcal{L}$. It is easy to check that $\psi$ is a continuous map and if we let $T$ act on $Y$ according to the formula $t f_{x}(s)=f_{x}(s t)$, then $t \psi(x)=t f_{x}=f_{t x}=\psi(t x)$. In fact we have:

LEMma 5.4. The action of $T$ on $Y$ is jointly continuous and the map $\psi:(X, T) \rightarrow(Y, T)$ is a homomorphism of T-systems.

6. The universal minimal $G$-system. Recall that the system of clopen subgroups $H_{n}=\left\{g \in G: g\left(I_{j}^{n}\right)=I_{j}^{n}, \forall 1 \leq j \leq 2^{n}\right\}, n=1,2, \ldots$, forms a basis for the topology of $G$ at the identity $e \in G$. By Theorem 5.3 it follows that the universal minimal dynamical system $(M(G), G)$ is zerodimensional. Let $D \subset M(G)$ be a clopen subset and $F_{D}=2 \mathbf{1}_{D}-\mathbf{1} \in C(M)$, with $\mathbf{1}_{D}$ the indicator function of $D$. If $H=\{g \in G: g D=D\}$ then $H$ is a clopen subgroup of $G$ and hence it contains $H_{n}$ for some $n$. It follows that the map $\psi_{D}$ constructed above (see the paragraph preceding Lemma 5.4) using $F_{D}$ as $F$ can be defined as a mapping into $\{1,-1\}^{H_{n} \backslash G}$ and thus we have $\psi_{D}:(M, G) \rightarrow\left(\Omega^{2^{n}}, G\right)$, so that with $Y_{D}=\psi_{D}(M(G))$, the system $\left(Y_{D}, G\right)$ is a minimal symbolic subsystem of $\Omega^{2^{n}}$.

Theorem 6.1. $(\Phi, G)$ is the universal minimal G-system. Hence all minimal actions of $G$ are proximal.

Proof. Since $(M(G), G)$ is the universal $G$-minimal system, there exists a homomorphism $\pi: M(G) \rightarrow \Phi$ and we pick some $m_{0} \in M(G)$ with $\pi\left(m_{0}\right)=c_{0}$ as a distinguished point for $M(G)$. 
Given a clopen subset $D \subset M(G)$ consider the following diagram:

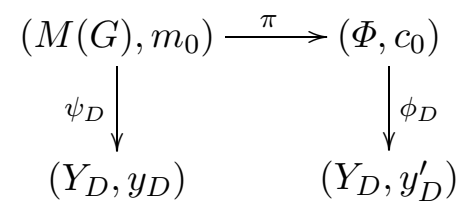

The homomorphism $\psi_{D}$ was defined above (see the first paragraph of the section) and $y_{D}=\psi_{D}\left(m_{0}\right)$. We apply Theorem 4.1 to define the homomorphism $\phi_{D}: \Phi \rightarrow \Omega^{k}$, with $y_{D}^{\prime}$ defined to be $\phi_{D}\left(c_{0}\right)$.

Now the image $\left(\psi_{D} \times\left(\phi_{D} \circ \pi\right)\right)\left(M(G), m_{0}\right)=\left(W,\left(y_{D}, y_{D}^{\prime}\right)\right)$, with $W \subset$ $Y_{D} \times Y_{D}$, is a minimal subset of the product system $\left(Y_{D} \times Y_{D}, G\right)$. Since the system $\left(Y_{D}, G\right)$ is proximal the diagonal $\Delta=\left\{(y, y): y \in Y_{D}\right\}$ is the unique minimal subset of the product system and we conclude that $y_{D}=y_{D}^{\prime}$, so that the above diagram is replaced by

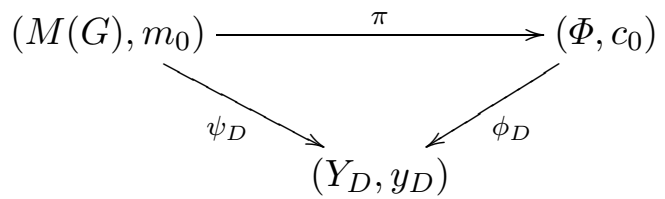

Next form the product space

$$
\Pi=\prod\left\{Y_{D}: D \text { a clopen subset of } M(G)\right\},
$$

and let $\psi: M(G) \rightarrow \Pi$ be the map whose $D$-projection is $\psi_{D}$ (i.e. $(\psi(m))_{D}$ $\left.=\psi_{D}(m)\right)$. We set $Y=\psi(M(G))$ and observe that since clearly the maps $\psi_{D}$ separate points on $M(G)$, the map $\psi: M(G) \rightarrow Y$ is an isomorphism, with $\psi\left(m_{0}\right)=y_{0}$, where $y_{0} \in Y$ is defined by $\left(y_{0}\right)_{D}=y_{D}$. Likewise define $\phi: \Phi \rightarrow Y$ by $(\phi(m))_{D}=\phi_{D}(m)$, so that also $\phi\left(c_{0}\right)=y_{0}$. These equations force the identity $\psi=\phi \circ \pi$ in the diagram

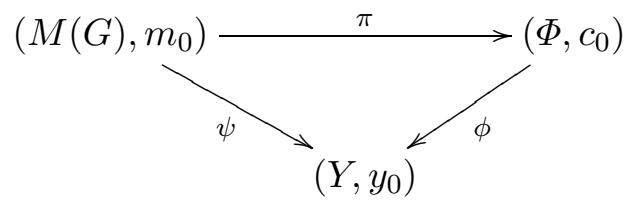

Since $\psi$ is a bijection it follows that so are $\pi$ and $\phi$, and the proof is complete.

\section{References}

[1] R. Ellis, Lectures on Topological Dynamics, W. A. Benjamin, New York, 1969.

[2] L. Gillman and M. Jerison, Rings of Continuous Functions, Van Nostrand, Princeton, 1960. 
[3] E. Glasner and B. Weiss, Minimal actions of the group $\mathbb{S}(\mathbb{Z})$ of permutations of the integers, Geom. Funct. Anal. 12 (2002), 964-988.

[4] R. L. Graham and B. Rothschild, Ramsey's theorem for n-parameter sets, Trans. Amer. Math. Soc. 159 (1971), 257-292.

[5] M. G. Megrelishvili and T. Scarr, The equivariant universality and couniversality of the Cantor cube, Fund. Math. 167 (2001), 269-275.

[6] V. G. Pestov, On free actions, minimal flows, and a problem by Ellis, Trans. Amer. Math. Soc. 350 (1998), 4149-4165.

[7] -, Remarks on actions on compacta by some infinite-dimensional groups, in: Infinite Dimensional Lie Groups in Geometry and Representation Theory (Washington, DC, 2000), World Sci., River Edge, NJ, 2002, 145-163.

[8] H. J. Prömel and B. Voigt, Graham-Rothschild parameter sets, in: Mathematics of Ramsey Theory, Springer, Berlin, 1990, 113-149.

[9] V. Uspenskij, On universal minimal compact G-spaces, Proc. 2000 Topology and Dynamics Conf. (San Antonio, TX), Topology Proc. 25 (2000), 301-308.

Department of Mathematics

Tel Aviv University

Ramat Aviv, Israel

E-mail: glasner@math.tau.ac.il
Institute of Mathematics Hebrew University of Jerusalem Jerusalem, Israel E-mail: weiss@math.huji.ac.il

Received 19 November 2002 